\title{
CONSISTENT PILOT DESIGNS FOR FREQUENCY OFFSET ESTIMATION IN MIMO OFDM SYSTEMS
}

\author{
Yinghui Li and Hlaing Minn \\ Department of Electrical Engineering, University of Texas at Dallas \\ Email: \{yinghui.li, hlaing.minn\}@utdallas.edu)
}

\begin{abstract}
This paper presents pilot designs for consistent frequency offset estimation of MIMO OFDM systems in frequency-selective fading channels. We derive the sufficient consistency condition for the pilots in MIMO OFDM systems to yield unambiguous estimation, and present corresponding consistent pilot designs. We develop pilot designs which satisfy both consistency over a limited CFO estimation range and the optimal channel estimation condition in MIMO OFDM systems. Simulation results corroborate that the consistent pilot design condition is efficient in choosing pilot patterns yielding better frequency offset estimation performance.
\end{abstract}

Index Terms - Frequency offset, MIMO OFDM, Consistency, Pilot design, Maximum likelihood estimation

\section{INTRODUCTION}

The carrier frequency offset (CFO) estimation plays an important role in orthogonal frequency-division multiplexing (OFDM) systems. Although crucial for emergency and other critical wireless systems, the problem of ambiguity of the CFO estimation metric trajectory within the considered estimation range has been overlooked until recently. Some research works on this problem for single input single output (SISO) OFDM systems have been discussed in [1] [2] for null tone based estimators and in [3] [4] for pilot based estimators, and several efficient consistent pilot design patterns have been proposed in these works.

All of the above existing pilot designs address only for SISO OFDM systems. Since consistency ${ }^{1}$ of CFO estimator are also crucial for realizing the advantages of Multiple input multiple output (MIMO) OFDM systems, in this paper we pursue these consistent pilot design issues for MIMO OFDM systems. We derive the sufficient condition for the consistent CFO estimation in MIMO OFDM systems using the estimators from [6] (the maximum likelihood estimator (MLE)) and [3], and develop corresponding consistent pilot design patterns for MIMO OFDM systems. Then we present pilot designs which satisfy consistent CFO estimation conditions as well as the optimal channel estimation condition. Simulation results corroborate the effectiveness of our pilot designs.

Notations: A bold small (capital) letter represents a column vector (matrix). The superscripts $*, T$, and $H$ represent the conjugate, the transpose, and the conjugate transpose operations, respectively. $[\boldsymbol{Y}]_{k, m}$ denotes the $k$-th row, $m$-th column element of $\boldsymbol{Y}$. All indices start from $0 . \boldsymbol{\Phi}[0: m, 0: n]$ denotes the sub-matrix of $\boldsymbol{\Phi}$ compromising of the first $m+1$ rows and the first $n+1$ columns of $\boldsymbol{\Phi}$. The all-one (all-zero) column vector of length- $k$, the $k \times m$ all-zero matrix, and the $k \times k$ identity matrix are denoted by $\mathbf{1}_{k}\left(\mathbf{0}_{k}\right), \mathbf{0}_{k \times m}$, and $\boldsymbol{I}_{k}$, respectively. The $i$-th column of $\boldsymbol{I}_{N}$

${ }^{1}$ The traditional definition of "consistency" in estimation implies that the estimation error tends to zero when the number of the observation samples tends to infinity. Our usage of consistency in this paper implies that the estimation yields an unbiased, ambiguity-free estimate under noise-free conditions regardless the channel response. is denoted by $\boldsymbol{e}_{i}$ and $\operatorname{diag}\{\boldsymbol{x}\}$ represents a diagonal matrix whose diagonal elements are defined by $\boldsymbol{x}$. The $l$-cyclic-shifted version of $c$ is denoted by $c^{(l)}$. $\otimes$ denotes the Kronecker product. $l \bmod N$ represents $l$ modulo $N .\lfloor X\rfloor$ denotes the integer part of $X$ while $\lceil X\rceil$ represents the smallest integer greater than or equal to $X . F$ denotes the $N$-point unitary discrete Fourier transform matrix and $\boldsymbol{f}_{k}$ is the $k$-th column of $\boldsymbol{F}$.

\section{SIGNAL MODEL AND FREQUENCY OFFSET ESTIMATION}

Consider a MIMO OFDM system where training signals are transmitted from $N_{t}$ transmit antennas to $N_{r}$ receive antennas over one OFDM symbol which contains $N$ sub-carriers. We assume that the channel impulse response (CIR) vector for each transmit-receive antenna pair has $L$ taps and is quasi-static over one OFDM symbol. Let $\boldsymbol{c}_{m}=\left[c_{m}(0), \ldots, c_{m}(N-1)\right]^{T}$ be the pilot tone vector of the $m$-th transmit antenna. Denote the indices of non-zero pilot tones and null tones of the $m$-th transmit antenna by $\left\{t_{m}(k): k=\right.$ $\left.0, \ldots, P_{m}-1\right\}$ and $\left\{n_{m}(k): k=0, \ldots, N-P_{m}-1\right\}$, where $P_{m}$ denotes the number of non-zero pilots on the $m$-th transmit antenna.

Let $\boldsymbol{s}_{m}=\left[s_{m}\left(-N_{g}\right), s_{m}\left(-N_{g}+1\right) \ldots, s_{m}(N-1)\right]$ denote the corresponding time-domain complex baseband training samples of the $m$-th transmit antenna, including $N_{g}(\geq L-1)$ cyclic prefix samples. Define $\boldsymbol{S}_{m}$ as the training signal matrix of size $N \times L$ for the $m$-th transmit antenna with elements given by $\left[\boldsymbol{S}_{m}\right]_{k, l}=$ $s_{m}(k-l)$ for $k \in\{0, \ldots, N-1\}$ and $l \in\{0, \ldots, L-1\}$. Let $\boldsymbol{h}_{(k, l)}$ denote the length- $L$ sample-spaced channel tap gain vector between the $k$-th transmit and $l$-th receive antenna pair.

After removing the cyclic prefix, the received vector $r$ from all $N_{r}$ receive antennas in the presence of a normalized (by the subcarrier spacing) frequency offset $v$ can be expressed as

$$
\begin{aligned}
\boldsymbol{r} & =\left[\boldsymbol{I}_{N_{r}} \otimes(\boldsymbol{\Gamma}(v) \boldsymbol{S})\right] \boldsymbol{h}+\boldsymbol{w} \\
\text { where } \boldsymbol{r} & =\left[\boldsymbol{r}_{0}^{T}, \ldots, \boldsymbol{r}_{N_{r}-1}^{T}\right]^{T} \\
\boldsymbol{\Gamma}(v) & =\operatorname{diag}\left\{1, e^{j 2 \pi v / N}, \ldots, e^{j 2 \pi(N-1) v / N}\right\} \\
\boldsymbol{S} & =\left[\boldsymbol{S}_{0}, \ldots, \boldsymbol{S}_{N_{t}-1}\right] \\
\boldsymbol{h} & =\left[\boldsymbol{h}_{(0,0)}^{T}, \boldsymbol{h}_{(1,0)}^{T}, \ldots, \boldsymbol{h}_{\left(N_{t}-1, N_{r}-1\right)}^{T}\right]^{T} \\
\boldsymbol{w} & =\left[\boldsymbol{w}_{0}^{T}, \boldsymbol{w}_{1}^{T}, \ldots, \boldsymbol{w}_{N_{r}-1}^{T}\right]^{T} .
\end{aligned}
$$

Here $\boldsymbol{r}_{k}$ is the $N \times 1$ received signal vector at the $k$-th receive antenna, and the elements of $\boldsymbol{w}$ are independent and identically distributed zero-mean circularly-symmetric complex Gaussian noise samples with variance $\sigma_{n}^{2}=E\left[\left|w_{i}(k)\right|^{2}\right] . S$ is a $N \times N_{t} L$ matrix. Applying the MLE from [6] to the MIMO system gives the MLE 
of $v$ as

$$
\begin{aligned}
\hat{v} & =\underset{\tilde{v}}{\arg \max }\{g(\tilde{v})\} \\
\text { where } g(\tilde{v}) & =\boldsymbol{r}^{H}\left(\boldsymbol{I}_{N_{r}} \otimes \boldsymbol{\Gamma}(\tilde{v}) \boldsymbol{B} \boldsymbol{\Gamma}^{H}(\tilde{v})\right) \boldsymbol{r} \\
\boldsymbol{B} & =\boldsymbol{S}\left(\boldsymbol{S}^{H} \boldsymbol{S}\right)^{-1} \boldsymbol{S}^{H} .
\end{aligned}
$$

If we apply the CFO estimator from [3] to MIMO systems, the resulting CFO estimator is given by (7) with

$$
\begin{aligned}
& g(\tilde{v})=\boldsymbol{r}^{H}\left(\boldsymbol{I}_{N_{r}} \otimes \boldsymbol{\Gamma}(\tilde{v}) \boldsymbol{B}_{2} \boldsymbol{\Gamma}^{H}(\tilde{v})\right) \boldsymbol{r} \\
& \text { where } \quad \boldsymbol{B}_{2}=\tilde{\boldsymbol{F}}_{P}^{*} \tilde{\boldsymbol{F}}_{P}^{T} \\
& \tilde{\boldsymbol{F}}_{P}=\left[\boldsymbol{f}_{t_{0}(0)}, \ldots, \boldsymbol{f}_{t_{0}\left(P_{0}-1\right)}, \boldsymbol{f}_{t_{1}(0)}, \ldots, \boldsymbol{f}_{t_{1}\left(P_{1}-1\right)},\right. \\
& \left.\quad \boldsymbol{f}_{t_{N_{t}-1}(0)}, \ldots, \boldsymbol{f}_{t_{N_{t}-1}\left(P_{N_{t}-1}-1\right)}\right] .
\end{aligned}
$$

\section{THE CFO ESTIMATOR CONSISTENCY CONDITION IN MIMO SYSTEMS}

The CFO estimation consistency condition for MIMO systems can be stated similar to the condition for SISO systems [4] as follows: "In the absence of noise, there is only one $\tilde{v}$ that maximizes the estimation metric $g(\tilde{v})$ and it is at $\tilde{v}=v$ for any $\boldsymbol{h} \neq \mathbf{0}$." Define

$$
G(\Delta)=g(v)-g(\tilde{v})
$$

where $\Delta=v-\tilde{v}$ and the range of $\Delta$ is $(v-N / 2, v+N / 2)$. Then the consistency condition stated above can be expressed as:

$$
G(\Delta)=0 \text { if and only if } \Delta=0, \forall \boldsymbol{h} \neq \mathbf{0} .
$$

For the MLE, substituting (8) into (13), we obtain

$$
G(\Delta)=\boldsymbol{h}^{H}\left[\boldsymbol{I}_{N_{r}} \otimes\left(\boldsymbol{S}^{H}\left(\boldsymbol{I}_{N}-\Gamma^{H}(\Delta) \boldsymbol{B} \boldsymbol{\Gamma}(\Delta)\right) \boldsymbol{S}\right)\right] \boldsymbol{h} .
$$

By singular value decomposition, we have $\boldsymbol{S}=\boldsymbol{U} \boldsymbol{\Sigma}_{\boldsymbol{S}} \boldsymbol{V}^{H}$ where $\boldsymbol{U}=\left[\boldsymbol{u}_{0}, \boldsymbol{u}_{1}, \ldots, \boldsymbol{u}_{N-1}\right]$ and $\boldsymbol{V}$ are the unitary matrices containing the eigen-vectors of $\boldsymbol{S} \boldsymbol{S}^{H}$, respectively. $\boldsymbol{\Sigma}_{\boldsymbol{S}}$ is the $N \times N_{t} L$ diagonal matrix with non-increasing singular values of $\boldsymbol{S}$. Here we assume $N \geq N_{t} L$ and $\boldsymbol{S}$ is a full-rank matrix, i.e. $\operatorname{rank}(\boldsymbol{S})=$ $N_{t} L$, which is not difficult to satisfy if the training sequence is designed appropriately. Note that the full rank condition for $S$ is required for identifiability of the joint MLE of CFO and CIR. Then we can express (9) as

$$
\begin{aligned}
& \boldsymbol{B}=\boldsymbol{U} \boldsymbol{\Sigma}_{\boldsymbol{S}}\left(\boldsymbol{\Sigma}_{\boldsymbol{S}}{ }^{H} \boldsymbol{\Sigma}_{\boldsymbol{S}}\right)^{-1} \boldsymbol{\Sigma}_{\boldsymbol{S}}{ }^{H} \boldsymbol{U}^{H}=\boldsymbol{U} \boldsymbol{\Sigma}_{\boldsymbol{B}} \boldsymbol{U}^{H} \\
& \text { where } \boldsymbol{\Sigma}_{\boldsymbol{B}}=\operatorname{diag}\left\{\mathbf{1}_{N_{t} L}^{T}, \mathbf{0}_{N-N_{t} L}^{T}\right\} .
\end{aligned}
$$

Substituting (16) into (15), we obtain

$$
\begin{aligned}
G(\Delta) & =\boldsymbol{z}^{H}(\Delta) \boldsymbol{z}(\Delta) \\
\boldsymbol{z}(\Delta) & =\left[\boldsymbol{I}_{N_{r}} \otimes\left(\left(\boldsymbol{I}-\boldsymbol{\Sigma}_{\boldsymbol{B}}\right) \boldsymbol{U}^{H} \boldsymbol{\Gamma}(\Delta) \boldsymbol{U} \boldsymbol{\Sigma}_{\boldsymbol{S}} \boldsymbol{V}^{H}\right)\right] \boldsymbol{h} .
\end{aligned}
$$

To satisfy the consistency condition in (14), we require that $\boldsymbol{z}(\Delta)=\mathbf{0}_{N N_{r}}$ only at $\Delta=0$ for any $|\tilde{v}|<N / 2$. In other words, we need $\boldsymbol{z} \neq \mathbf{0}_{N N_{r}}$ for any $|\hat{v}|<N / 2$ except $\hat{v}=v$. By using (19), this condition can be achieved for any $\boldsymbol{h} \neq \mathbf{0}_{N_{r} N_{t} L}$ if $\boldsymbol{I}_{N_{r}} \otimes\left(\left(\boldsymbol{I}-\boldsymbol{\Sigma}_{\boldsymbol{B}}\right) \boldsymbol{U}^{H} \boldsymbol{\Gamma}(\Delta) \boldsymbol{U} \boldsymbol{\Sigma}_{\boldsymbol{S}} \boldsymbol{V}^{H}\right)$ is a full-rank matrix. Then, the consistency condition can be achieved for any $\boldsymbol{h} \neq \mathbf{0}$ if the following necessary and sufficient condition is satisfied:

$$
\begin{aligned}
\operatorname{rank}\left(\boldsymbol{I}_{N_{r}} \otimes\left(\left(\boldsymbol{I}-\boldsymbol{\Sigma}_{\boldsymbol{B}}\right) \boldsymbol{U}^{H} \boldsymbol{\Gamma}(\Delta) \boldsymbol{U} \boldsymbol{\Sigma}_{\boldsymbol{S}} \boldsymbol{V}^{H}\right)\right)= & N_{r} N_{t} L, \\
& \forall \Delta \neq 0 .
\end{aligned}
$$

By using (17), together with $\operatorname{rank}(\boldsymbol{S})=N_{t} L$, assuming that every transmit-receive antenna pair has the same CIR length $L$, we obtain from (20) a sufficient condition for consistency as

$$
\begin{gathered}
\operatorname{rank}(\boldsymbol{S})=N_{t} L \& \operatorname{rank}\left(\boldsymbol{U}_{2}^{H} \boldsymbol{\Gamma}(\Delta) \boldsymbol{U}_{1}\right)=N_{t} L, \forall \Delta \neq 0 \\
\text { where } \boldsymbol{U}_{1}=\left[\boldsymbol{u}_{0}, \boldsymbol{u}_{1}, \ldots, \boldsymbol{u}_{N_{t} L-1}\right] \\
\boldsymbol{U}_{2}=\left[\boldsymbol{u}_{N_{t} L}, \boldsymbol{u}_{N_{t} L+1}, \ldots, \boldsymbol{u}_{N-1}\right] .
\end{gathered}
$$

Similarly, we obtain the following sufficient condition for the consistency of the CFO estimator in [3]:

$\operatorname{rank}\left(\left(\sum_{m=0}^{N_{t}-1}\left(\boldsymbol{I}_{N}-\boldsymbol{\Sigma}_{C_{m}}\right)\right) \boldsymbol{F} \boldsymbol{\Gamma}(\Delta) \tilde{\boldsymbol{F}}^{H} \boldsymbol{C}\left(\boldsymbol{I}_{N_{t}} \otimes \boldsymbol{F}_{L}\right)\right)=N_{t} L$.

$$
\text { where } \quad \begin{aligned}
\boldsymbol{F}_{L} & =\left[\boldsymbol{f}_{0}, \boldsymbol{f}_{1}, \ldots, \boldsymbol{f}_{L-1}\right] \\
\tilde{\boldsymbol{F}} & =\mathbf{1}_{N_{t}} \otimes \boldsymbol{F} \\
\boldsymbol{C}_{m} & =\operatorname{diag}\left\{\boldsymbol{c}_{m}\right\} \\
\boldsymbol{C} & =\operatorname{diag}\left\{\boldsymbol{C}_{0}, \ldots, \boldsymbol{C}_{N_{t}-1}\right\} \\
\boldsymbol{\Sigma}_{C_{m}} & =\boldsymbol{C}_{m}\left(\boldsymbol{C}_{m}^{H} \boldsymbol{C}_{m}\right)^{-1} \boldsymbol{C}_{m}^{H} . \\
\boldsymbol{\Sigma}_{C} & =\boldsymbol{C}\left(\boldsymbol{C}^{H} \boldsymbol{C}\right)^{-1} \boldsymbol{C}^{H} .
\end{aligned}
$$

\section{PROPOSED CONSISTENT PILOT DESIGNS}

\section{4-A. MLE Consistent Pilot Design Condition}

Denote the union of the non-zero pilot tones indices over $N_{t}$ transmit antennas by $\{t(k): k=0, \ldots, P-1\}=\bigcup_{m=0}^{N_{t}-1}\left\{t_{m}(k)\right.$ : $\left.k=0, \ldots, P_{m}-1\right\}$, and the intersection of the null pilot tones indices over $N_{t}$ transmit antennas by $\{n(k): k=0, \ldots, N-P-$ $1\}=\bigcap_{m=0}^{N_{t}-1}\left\{n_{m}(k): k=0, \ldots, N-P_{m}-1\right\}$, respectively. Note that $\{t(k): k=0, \ldots, P-1\} \bigcup\{n(k): k=0, \ldots, N-P-1\}=$ $\{0, \ldots, N-1\}$. Define

$$
\begin{aligned}
t^{(l)}(k)=(t(k)+l) \bmod N, \quad l \in\{0,1,2, \ldots, N-1\} & \\
X= & \left\{t^{(l)}(k): k=0, \ldots, P-1\right\} \bigcap\{n(k): k=0, \ldots, \\
& N-P-1\} \\
\boldsymbol{Z}= & {\left[\boldsymbol{I}_{P}, \mathbf{0}_{P \times(N-P)}\right] } \\
\boldsymbol{\Theta}= & {\left[\boldsymbol{e}_{t(0)}, \boldsymbol{e}_{t(1)}, \ldots, \boldsymbol{e}_{t(P-1)}, \boldsymbol{e}_{n(0)}, \boldsymbol{e}_{n(1)} \ldots, \boldsymbol{e}_{n(N-P-1)}\right] } \\
\boldsymbol{D}= & \boldsymbol{Z} \boldsymbol{\Theta}^{T}\left[\boldsymbol{C}_{0} \boldsymbol{F}_{L}, \boldsymbol{C}_{1} \boldsymbol{F}_{L}, \ldots, \boldsymbol{C}_{N_{t}-1} \boldsymbol{F}_{L}\right]
\end{aligned}
$$

Then, for a MIMO OFDM system with $N_{t}$ transmit antennas and length- $L$ CIR vectors of all transmit-receive antenna pairs, a sufficient pilot design condition for the consistency of the MLE is comprised of the following two parts:

Part 1. The design condition to choose the locations of the consistent pilots over all transmit antennas: " $(N-P) \geq P$, $P>N_{t} L, \operatorname{rank}(\boldsymbol{S})=N_{t} L$, and for any cyclic-shifting distance $l \in\{1,2, \ldots, N-1\}$ for the full range CFO estimation, or $l \in\{-\lfloor\Omega / 2\rfloor, \ldots,-1,1, \ldots, \mid \Omega / 2\rfloor-1\}(\Omega \leq N)$ for the limited range $C F O$ estimation, the cardinality of the set $X$ defined in (32) is always larger than or equal to $N_{t} L$."

Part 2. The design condition to arrange the locations and values of the pilots on each transmit antenna: “ $\boldsymbol{D}_{K}$ is always a full-rank matrix, i.e. $\operatorname{rank}\left(\boldsymbol{D}_{K}\right)=N_{t} L$, where $\boldsymbol{D}_{K}$ denotes the matrix formed by any $K\left(N_{t} L \leq K \leq P\right)$ rows of $\boldsymbol{D}$ in (35)."

If both of the above conditions are satisfied, the designed pilots are guaranteed to be consistent. The proof is omitted due to space limitation. 
Let $\boldsymbol{c}$ denote the length- $N$ (virtual pilot) vector whose indices of non-zero pilot tones and null tones are the same as $\{t(k): k=$ $0, \ldots, P-1\}$ and $\{n(k): k=0, \ldots, N-P-1\}$, respectively, where $P$ is the number of non-zero pilot tones of $c$. Then the sufficient consistent pilot design condition can be rewritten as:

Part 1. " $(N-P) \geq P, P>N_{t} L, \operatorname{rank}(\boldsymbol{S})=N_{t} L$, and for any cyclic-shifting distance $l \in\{1,2, \ldots, N-1\}$ for the full range $C F O$ estimation, or $l \in\{-\lfloor\Omega / 2\rfloor, \ldots,-1,1, \ldots,\lfloor\Omega / 2\rfloor-1\}$ for the limited range CFO estimation, $c^{(l)}$ always has at least $N_{t} L$ non-zero pilot tones located at the null-tone indices of the original unshifted virtual pilot vector c."

Part 2. "rank $\left(\boldsymbol{D}_{K}\right)=N_{t} L$ for any $K$ where $N_{t} L \leq K \leq P$."

We can relax the consistent pilot design condition by introducing the consistency in the probabilistic sense where there can be some $\boldsymbol{h}$ which yield inconsistency but the probability of their occurrence is zero. The details are omitted due to space limitation.

\section{4-B. Consistent Pilot Designs}

In the following, we will only discuss for the full-range consistent pilot design since this process can be easily extended for an arbitrary limited estimation range. According to the two parts of the consistent pilot design condition proposed above, we separate the consistency pilot design procedure for MIMO OFDM systems into two steps accordingly:

Step 1) The design of $\boldsymbol{c}$, the virtual pilot vector with $P\left(>N_{t} L\right)$ non-zero pilot tones, for the consistency in MIMO OFDM systems:

This task is very similar to the pilot design we proposed in [4] and [5] for the SISO systems. Several efficient consistent pilot design patterns for the full-range $\mathrm{CFO}$ estimation were introduced in [4] based on different code structures, such as BCH codes, Cyclic codes, m-sequence, distinctive-spacing, etc. Furthermore, the consistent pilot designs for an arbitrary limited CFO estimation range are proposed in [5]. We can choose any of them as our $c$ according to the CFO estimation range of interest.

Step 2) The design of the pilot vector $\boldsymbol{c}_{m}$ on each $m$-th transmit antenna, satisfying $\bigcup_{m=0}^{N_{t}-1}\left\{t_{m}(k): k=0, \ldots, P_{m}-1\right\}=\{t(k)$ : $k=0, \ldots, P-1\}, \operatorname{rank}(\boldsymbol{S})=N_{t} L$ and $\operatorname{rank}\left(\boldsymbol{D}_{K}\right)=N_{t} L$ for any $K$ where $N_{t} L \leq K \leq P$ :

The design patterns to assign pilot tones over $N_{t}$ transmit antennas are not unique. In the following, we present two patterns while the proof for their validity is omitted due to space limitation.

Pattern (a): Construct a Vandermonde matrix $\boldsymbol{\Psi}$ with the $(k, m)$ th element defined by

$$
[\Psi]_{k, m}=e^{j m \theta_{k}}
$$

where $\theta_{k} \neq \theta_{n}, \forall k \neq n$. Note that we already have the virtual pilot vector $c$ with $P$ non-zero pilot tones from Step 1. Choosing an arbitrary integer $J$ satisfying $\lceil P / L\rceil \leq J \leq P$ and defining $Q=\lfloor P / J\rfloor$, we construct a $P \times N_{t}$ matrix $\boldsymbol{\Xi}_{1}$ as

$\boldsymbol{\Xi}_{1}=\left[\boldsymbol{e}_{\lfloor 0 / Q\rfloor}, \boldsymbol{e}_{\lfloor 1 / Q\rfloor}, \ldots, \boldsymbol{e}_{\lfloor(P-1) / Q\rfloor}\right]^{T} \boldsymbol{\Psi}\left[0:(J-1), 0:\left(N_{t}-1\right)\right]$.

Then, the pilot tones for the $m$-th $\left(0 \leq m \leq N_{t}-1\right)$ transmit antenna are given by

$$
\boldsymbol{c}_{m}[t(k)]=\boldsymbol{c}[t(k)]\left[\boldsymbol{\Xi}_{1}\right]_{k, m} \quad \& \quad \boldsymbol{c}_{m}[n(k)]=0 .
$$

An alternative form of Pattern (a) is given by

$$
\boldsymbol{c}_{m}[t(k)]=\boldsymbol{c}[t(k)] e^{j m \theta_{\lfloor k / J\rfloor}} \quad \& \quad \boldsymbol{c}_{m}[n(k)]=0 .
$$

Pattern (b): We construct a $P \times N_{t}$ matrix $\boldsymbol{\Xi}_{2}$ as

$$
\begin{gathered}
\boldsymbol{\Xi}_{2}=\left[\boldsymbol{e}_{\lfloor 0 / Q\rfloor}, \boldsymbol{e}_{\lfloor 1 / Q\rfloor}, \ldots, \boldsymbol{e}_{\lfloor(P-1) / Q\rfloor}\right]^{T}\left[\boldsymbol{I}_{N_{t}},\right. \\
\left.\boldsymbol{\Psi}\left[0:\left(J-N_{t}-1\right), 0:\left(N_{t}-1\right)\right]^{T}\right]^{T} .
\end{gathered}
$$

Then the pilot tones for the $m$-th transmit antenna are given by

$$
\boldsymbol{c}_{m}[t(k)]=\boldsymbol{c}_{m}[t(k)]\left[\boldsymbol{\Xi}_{2}\right]_{k, m} \quad \& \quad \boldsymbol{c}_{m}[n(k)]=0 .
$$

An alternative expression for $\boldsymbol{c}[t(k)]$ of Pattern (b) is given by

$\boldsymbol{c}_{m}[t(k)]= \begin{cases}\boldsymbol{c}[t(k)], & m Q \leq k<(m+1) Q \\ \boldsymbol{c}[t(k)] e^{j m \theta_{\left\lfloor\left(k-N_{t} Q\right) / J\right\rfloor},} & k \geq N_{t} Q \\ 0, & \text { else }\end{cases}$

The consistent pilot design condition for the MLE also satisfies that for the estimator in [3]. Consequently, all of the consistent pilot patterns proposed for the MLE as stated above also hold for the estimator in [3].

\section{4-C. Pilot Designs For Both Consistent CFO MLE and Optimal Channel Estimation}

The designed training sequences for CFO estimation may also be used to estimate the channels. The optimal training signals for the estimation of frequency-selective channels in MIMO OFDM systems [7] satisfy the following condition:

$$
\begin{aligned}
\boldsymbol{S}^{H} \boldsymbol{S} & =E_{\mathrm{av}} \boldsymbol{I} \\
\text { where } E_{\mathrm{av}} & =\frac{1}{N_{t}} \sum_{m=0}^{N_{t}-1} E_{m} \text { and } E_{m}=\sum_{k=0}^{N-1}\left|s_{m}(k)\right|^{2} .
\end{aligned}
$$

In [7], the pilot tone allocations among transmit antennas are classified as frequency division multiplexing (FDM), time division multiplexing (TDM), code division multiplexing in time-domain (CDM-T), code division multiplexing in frequency-domain (CDMF), and combinations thereof. In this paper we only consider one OFDM symbol, and hence the TDM and CDM-T allocations can not be used here. The FDM type violates Part 2 of the consistent pilot design condition we proposed in Section 4.A, and hence we will consider the consistent pilot designs based on the CDM-F type defined in [7].

For a limited CFO estimation range (smaller than the full range), it is possible to design pilots satisfying both the consistency condition in the limited CFO estimation range such as $\left(-\frac{N}{2 L}, \frac{N}{2 L}\right]$ and the optimal channel estimation condition. In our approach, we use periodic pilot allocation patterns to satisfy the optimal channel estimation condition and design a consistent pilot tones vector for one period of the whole training sequence on one antenna, and develop the pilot tones on different antennas according to the optimal pilot allocation for channel estimation while maintaining CFO estimation consistency over the considered range. In the following, we present the design patterns assuming that $N$ is a multiple of the CIR vector length $L$, i.e. $N=M L$.

CDM-F based design:

This design consists of the following two steps.

i) Design the length- $M$ consistent pilot tone subvector $\boldsymbol{d}$ ( $\boldsymbol{d}$ is consistent over the full-range of $(-M / 2, M / 2])$ according to the design described in Step 1 of Section 4.B, i.e. following the same consistent pilot design process in [4] and [5] to design a virtual consistent pilot sub-vector $\boldsymbol{d}$.

Denote the number of non-zero pilot tones of $\boldsymbol{d}$ by $P^{(d)}$ and the corresponding tone indices by $\left\{t(k)_{d}\right\}$. Then $\boldsymbol{d}$ satisfies

$$
\left|d\left[t(k)_{d}\right]\right|=d_{0}, \forall k, d_{0}>0 \quad \& \quad P^{(d)}>N_{t} L .
$$

Next, construct the virtual pilot vector $c$ by repeating $\boldsymbol{d} L$ times, i.e. $c=1_{L} \otimes \boldsymbol{d}$, and its $P^{(d)} L$ nonzero pilot tones' indices are denoted by $\{t(k)\}$ and the remaining $\left(N-P^{(d)} L\right)$ null tones' indices are denoted by $\{n(k)\}$. Hence, the virtual pilot vector $\boldsymbol{c}$ satisfies the consistency condition for the limited estimation range $v \in(-M / 2, M / 2]$.

ii) The pilot tones of the $m$-th $\left(0 \leq m \leq N_{t}-1\right)$ transmit antenna are given by one of the CDM-F pilot allocation patterns defined in [7]. An example design is as follows:

$$
\boldsymbol{c}_{m}[t(k)]=\boldsymbol{c}[t(k)] e^{\frac{-j 2 \pi m k}{P^{(d)}}} \quad \& \quad \boldsymbol{c}_{m}[n(k)]=0 .
$$


Note that we can also have other design patterns besides the above CDM-F based designs.

\section{SIMULATION RESULTS}

We consider a MIMO OFDM system with $N=64$ sub-carriers, $N_{t}$ transmit antennas and $N_{r}$ receive antennas in multipath Rayleigh fading channels with $L=4$ (unless otherwise stated) samplespaced taps having an exponential power delay profile with a $3 \mathrm{~dB}$ per tap decaying factor (unless otherwise stated). Simulation results are obtained from $10^{5}$ independent runs.

Fig. 1 and Fig. 2 illustrate the effect of inconsistent pilot design.

In Fig. 1, we present the estimation metric for an inconsistent pilot pattern which does not satisfy the consistent pilot design condition in Section 3. The multiple maxima of the estimation metric result in the failure in CFO estimation as we will show in the next figure.

Fig. 2 presents the CFO estimation mean-square error (MSE) comparison between the inconsistent pilot and other two consistent pilots for a fixed channel which yields inconsistency for the inconsistent pilot in Fig. 1. Due to this inconsistency, a complete estimation failure is observed for the inconsistent pilot.

Fig. 3 compares the uncoded BER performance in a BPSK MIMO OFDM (V-BLAST) system between two of the proposed pilot designs: i) the design satisfying both the CFO estimation consistency and the optimal channel estimation condition; ii) the design satisfying the CFO estimation consistency condition only. Both CFO and channel estimation use the same pilot signal and maximum likelihood detection is performed. Fig. 3 shows that our design with both CFO consistency and optimal channel estimation gives about $1 \mathrm{~dB}$ SNR advantage over our design with $\mathrm{CFO}$ consistency only.

\section{CONCLUSIONS}

Consistent CFO estimation is important in maintaining a reliable wireless link for any system especially emergency or other critical communication systems. For MIMO OFDM systems, we have developed sufficient conditions for the consistent pilot design of the $\mathrm{CFO}$ estimation and presented the corresponding pilot designs. We have also developed pilot designs which satisfy the CFO estimation consistency as well as the optimal channel estimation condition. Our proposed pilot design criteria for the consistency constitute a very efficient pilot design tool as corroborated by the simulation results.

Acknowledgment: This work is supported by the Erik Josson School Research Excellence Initiative, UTD.

\section{REFERENCES}

[1] X. Ma, et. al, "Non-data-aided carrier offset estimators for OFDM with null subcarriers: identifiability, algorithm, and performance," IEEE Journ. Select Areas in Commun., vol.19, no.12, pp. 2504-2515, Dec. 2001.

[2] H. Liu et. al, "A high efficiency estimator for OFDM communications," IEEE Commun. Lett., vol. 2, pp. 104-106, Apr. 1998.

[3] J. Lei et. al, "A consistent OFDM carrier frequency offset estimator based on distinctively spaced pilot tones," IEEE Trans. Wireless Commun., vol. 3, no. 2, pp. 588-599, Mar. 2004.

[4] H. Minn, et. al, "Pilot designs for consistent frequency offset estimation in OFDM systems", IEEE ICC, June 2006.

[5] Y. Li, et. al, "Robust pilot design for consistent carrier frequency offset estimation", accepted in Milcom 2006.

[6] M. Morelli et. al, "Carrier-frequency estimation for transmissions over selective channels," IEEE Trans. Commun., vol. 48, no. 9, pp. 1580-1589, Sept. 2000.

[7] H. Minn et. al, "Optimal training signals for MIMO OFDM channel estimation" IEEE Trans. Wireless Commun., vol. 5, no. 5, pp. 1158 - 1168, May 2006.

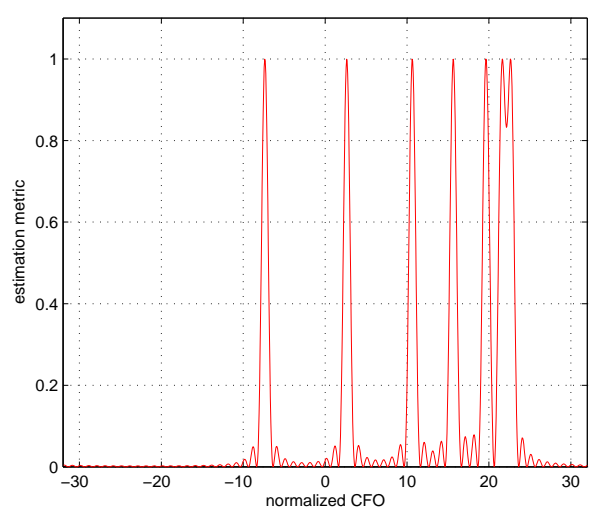

Fig. 1. The CFO estimation metric (normalized) associated with an inconsistent pilot for a MIMO OFDM system with $N=64$, $L=4, N_{t}=2$ and $N_{r}=2$

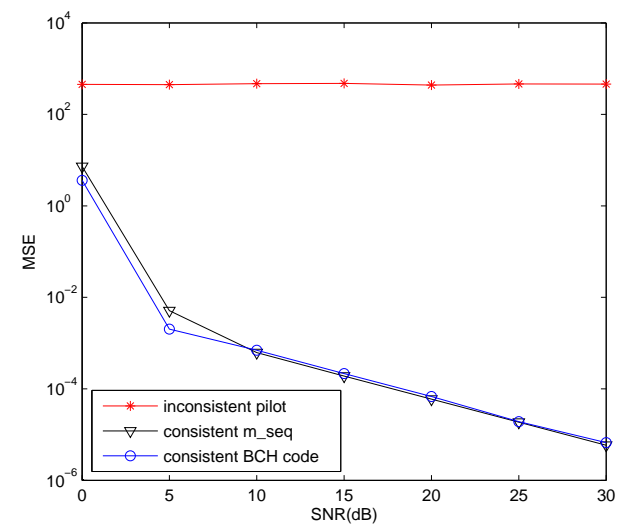

Fig. 2. The CFO estimation MSE performance associated with different preambles for a particular channel realization which yields inconsistency for a MIMO OFDM system with $N_{t}=2$ and $N_{r}=1$

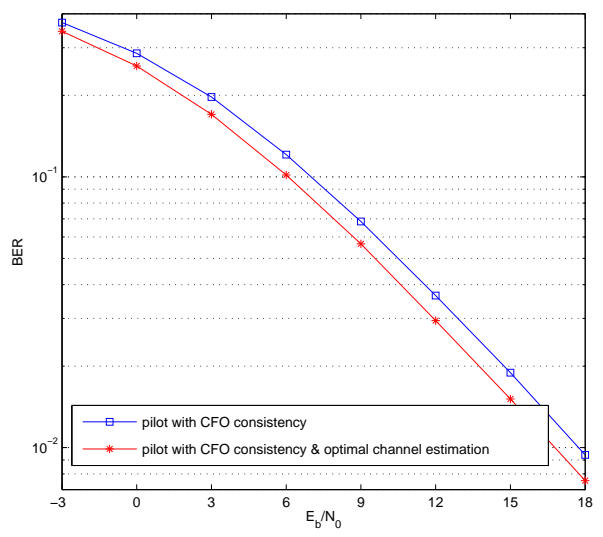

Fig. 3. The uncoded BER comparison between the proposed pilot design satisfying CFO estimation consistency and optimal channel estimation and the proposed pilot design satisfying CFO estimation consistency only, in a BPSK MIMO OFDM system (V-BLAST) with $N=64, L=2, N_{t}=2$ and $N_{r}=1$ 